Sādhanā Vol. 29, Part 5, October 2004, pp. 415-428. ( $)$ Printed in India

\title{
Free asymmetric transverse vibration of polar orthotropic annular sector plate with thickness varying parabolically in radial direction
}

\author{
R P SINGH and S K JAIN \\ Department of Applied Mechanics, Birla Institute of Technology, Mesra, \\ Ranchi 835 215, India \\ e-mail: rps_bit@sify.com; saralkjain@yahoo.com
}

MS received 30 August 2003; revised 27 January 2004

\begin{abstract}
In the present paper, free asymmetric transverse vibrations of a nonuniform polar orthotropic annular sector plate, with parabolically varying thickness in the radial direction, have been studied on the basis of classical plate theory. The circular edges of the plate are elastically restrained against translation and rotation while the straight (radial) edges are simply-supported. Fourth-order linear differential equations with variable coefficients governing the motion have been solved by using the collocation interpolation technique with Chebyshev points as the interpolating nodes. Frequency parameters of the plate with flexible boundary conditions at the circular edges for some typical values of the taper parameters, the rigidity ratios and the radii ratio have been presented.
\end{abstract}

Keywords. Asymmetric transverse vibration; polar orthotropic; annular sector plate; parabolically varying thickness; translational and rotational spring parameters; Chebyshev points.

\section{Introduction}

Plate-like structural elements of sectorial geometry have been widely used in many engineering applications, for example, in aircraft, missiles, naval structures, nuclear reactors etc. Transverse vibrations of uniform isotropic sector plates for symmetrical modes have earlier, been theoretically analysed by Rubin (1957), Ben-Amoz (1959) and Westmann (1962). Later on, Ramakrishnan \& Kununnasseril (1973), Ramaiah \& Vijaykumar (1974), Bhattacharya \& Bhowmic (1975) and Wilson \& Garg (1978) also studied isotropic sectorial plates with various circular edge conditions. Mizusawa (1991) applied spline elements in the study of annular sector plates. Recently, Liew \& Liu (2000) studied flexural vibrations of shear deformable isotropic annular sector plates by a differential quadrature method while Houmat (2001) studied such plates by applying the Fourier $p$-version of the finite element method.

A list of symbols is given at the end of the paper 
After the development of the high strength-to-weight ratio of fibre-reinforced composite materials exhibiting anisotropic characteristics, in the past few decades, much research has been done on the flexural vibration problems of polar orthotropic sectorial plates. To mention a few, Rubin $(1975,1978)$ studied free vibration and stability of polar orthotropic sector plates by a series solution. Irie et al $(1979,1988)$ treated the free vibrations of ring-shaped polar orthotropic sectorial plate by the Ritz method using a spline function as an admissible function for the deflection of plates. Mukhopadhyay $(1979,1982)$ also dealt with polar orthotropic sector plates by using a semi analytical method. Narita (1985) studied free vibrations of polar orthotropic sector plates with point support at an arbitrary location by the Lagrange multiplier method as an extension of the Ritz method.

Under these studies, free axisymmetric transverse vibration of isotropic/anisotropic sectorial plates of uniform thickness have been analysed mostly under classical boundary conditions (free, clamped, simply-supported). However, it is an accepted fact that the condition on a periphery often tends to be mid-way between classical boundary conditions and may correspond more closely to some form of elastic restraints. Many researchers have worked on flexible boundary conditions on the curved edges. To mention a few, Kim \& Dickinson (1990) have studied flectural vibrations of isotropic and polar orthotropic annular and circular plates with elastically restrained peripheries. Mukhopadhyay (1982) has studied free vibrations of annular sector plates with edges possessing different degrees of rotational restraint.

The development of fibre-reinforced composite materials with high strength-to-weight ratios and exhibiting anisotropic characteristics and their increasing use in modern industries have encouraged researchers to study free vibrations of non-uniform sector plates. A lot of research work has been done on free vibrations of non-uniform annular plates. A few such as Lal \& Gupta (1982) have studied axisymmetric vibrations of polar orthotropic annular plates of variable thickness, while Chen (1997) has studied axisymmetric vibrations of circular and annular plates with arbitrarily varying thicknesses. In the case of high speed turbo machinery, it is desirable to study free asymmetric transverse vibrations of such plates.

In an attempt to generalize the study of plate vibration, free asymmetric transverse vibrations of polar orthotropic non-uniform annular sector plates (parabolically varying thickness) along the radial direction have been studied. This has been done for straight (radial) edges when they are simply-supported while the curved edges are elastically restrained against translation and rotation. A simple collocation interpolation technique has been considered for computing the results and the effects of orthotropy, taper parameters and flexibility parameters on the frequency parameters of the plate. In our study on thin plate vibrations (Singh \& Jain 2001), we have shown that, instead of equi-spaced nodes, using Chebyshev collocation points as the interpolating nodes gives faster convergence and better results. Hence, in the present research paper also, Chebyshev points have been taken as the interpolating nodes.

\section{Analysis}

\subsection{Equation of motion}

Consider a thin annular sector plate of opening angle $\Phi$, inner radius $b$, outer radius $a$ with parabolically varying thickness $h(r)$ and with its circular edges elastically restrained against translation and rotation. Its straight (radial) edges are simply-supported as shown in figure 1 (centrally thinner case). Let $(r, \theta)$ be the polar co-ordinates on any point in the neutral surface referred to the centre of the plate as origin. Without taking the rotary inertia and shear deformation into account, the equation of motion of a harmonically vibrating polar orthotropic 


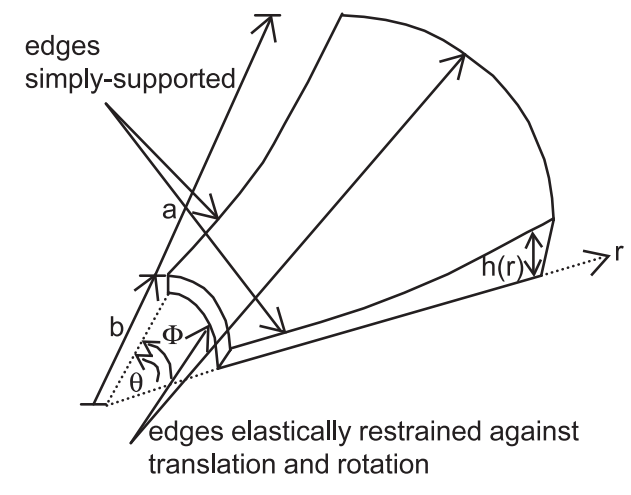

Figure 1. Non-uniform (centrally thinner) annular sector plate.

uniform thickness plate given by Lekhnitskii (1968) has been extended further for the nonuniform thickness case. After taking the transverse deflection $w(r, \theta)=a \Phi G(\xi) H(\eta)$ (after separation of variables and normalization), where $G(\xi)$ and $H(\eta)$ are functions of $\xi(=r / a)$ and $\eta(=\theta / \Phi)$ respectively and $H(\eta)=\sin n \pi \eta$ (Levy's problem), the equation of motion of polar orthotropic sectorial plate with radial edges as simply-supported has been expressed in non-dimensional form as:

$$
\begin{aligned}
& F(\xi) \frac{\mathrm{d}^{4} G(\xi)}{\mathrm{d} \xi^{4}}+\left(\frac{F_{1}}{\xi}\right) \frac{\mathrm{d}^{3} G(\xi)}{\mathrm{d} \xi^{3}}+\left(\frac{F_{2}}{\xi^{2}}\right) \frac{\mathrm{d}^{2} G(\xi)}{\mathrm{d} \xi^{2}} \\
& +\left(\frac{F_{3}}{\xi^{3}}\right) \frac{\mathrm{d} G(\xi)}{\mathrm{d} \xi}+\left(\frac{F_{4}}{\xi^{4}}\right) G(\xi)-\lambda^{2} f(\xi) G(\xi)=0,
\end{aligned}
$$

where

$$
\begin{aligned}
F_{1}= & 2\left\{\frac{\xi \mathrm{d} F(\xi)}{\mathrm{d} \xi+F(\xi)}\right\}, \\
F_{2}= & \frac{\xi^{2} \mathrm{~d}^{2} F(\xi)}{\mathrm{d} \xi^{2}+\left(2+v_{\theta}\right)} \frac{\xi \mathrm{d} F(\xi)}{\mathrm{d} \xi}-\left\{k^{2}+2\left(\frac{n \pi}{\Phi}\right)^{2}\left(v_{\theta}+2 D_{k r}\right)\right\} F(\xi), \\
F_{3}= & \xi^{2} v_{\theta} \frac{\mathrm{d}^{2} F(\xi)}{\mathrm{d} \xi^{2}-\xi}\left\{k^{2}+2\left(\frac{n \pi}{\Phi}\right)^{2}\left(v_{\theta}+2 D_{k r}\right)\right\} \frac{\mathrm{d} F(\xi)}{\mathrm{d} \xi} \\
& +\left\{k^{2}+2\left(\frac{n \pi}{\Phi}\right)^{2}\left(v_{\theta}+2 D_{k r}\right)\right\} F(\xi), \\
F_{4}= & -\xi^{2} v_{\theta}\left(\frac{n \pi}{\Phi}\right)^{2} \frac{\mathrm{d}^{2} F(\xi)}{\mathrm{d} \xi^{2}}+\xi\left(\frac{n \pi}{\Phi}\right)^{2}\left\{k^{2}+2\left(v_{\theta}+2 D_{k r}\right)\right\} \frac{\mathrm{d} F(\xi)}{\mathrm{d} \xi} \\
& +\left(\frac{n \pi}{\Phi}\right)^{2}\left\{\left(\frac{k n \pi}{\Phi}\right)^{2}-2 k^{2}-2\left(v_{\theta}+2 D_{k r}\right)\right\} F(\xi),
\end{aligned}
$$

where $f(\xi)$ is a thickness variation function. For a parabolically varying thickness (PVT), $f(\xi)=\left(1+\alpha \xi^{2}\right)$ where $\alpha$ is a taper parameter, $F(\xi)=f^{3}(\xi), D_{k r}=G_{r \theta}\left(1-v_{r} v_{\theta}\right) / E_{r}$, 
where $G_{r \theta}$ is shear modulus; $v_{r}, v_{\theta}$ are Poisson's ratios; $E_{r}, E_{\theta}$ are Young's modulus in radial and tangential direction, respectively and $k^{2}=E_{\theta} / E_{r}$. The non-dimensional frequency parameter, $\Omega=\lambda^{2}=\rho \omega^{2} a^{4} h_{b} / D_{r b}$, where $\rho$ is mass per unit volume, $\omega$ is circular frequency, $h_{b}$ is plate thickness at inner edge and $D_{r b}=E_{r} h_{b}^{3} / 12\left(1-v_{r} v_{\theta}\right)$, while $n$ is a positive integer $(1,2,3 \ldots)$ and $(n-1)$ gives the number of radial nodal lines.

\subsection{Boundary conditions}

2.2a When circular edges are elastically restrained against rotation: Bending moment per unit length required to cause rotation (Lessia 1969),

$$
M_{r}=-k_{r}(\partial w(r, \theta) / \partial r)
$$

where $k_{r}$ is rotational stiffness constant.

But from the moment-curvature relation (Lekhnitskii 1968)

$$
M_{r}=-D_{r}\left[\frac{\partial^{2} w(r, \theta)}{\partial r^{2}}+v_{\theta}\left\{\left(\frac{1}{r}\right) \frac{\partial w(r, \theta)}{\partial r}+\left(\frac{1}{r^{2}}\right) \frac{\partial^{2} w(r, \theta)}{\partial \theta^{2}}\right\}\right],
$$

where $D_{r}=E_{r} h^{3} / 12\left(1-v_{r} v_{\theta}\right)$ and $h=h(r)$ is the local thickness of the plate.

After normalization and further simplifications, (2) and (3) give:

$$
F(\varepsilon) \frac{\mathrm{d}^{2} G(\varepsilon)}{\mathrm{d} \xi^{2}}+\left(\frac{\left(v_{\theta} F(\varepsilon)-K_{r b}\right)}{\varepsilon}\right) \frac{\mathrm{d} G(\varepsilon)}{\mathrm{d} \xi}-\left(n^{2} \pi^{2} v_{\theta} \frac{F(\varepsilon)}{\Phi^{2}} \varepsilon^{2}\right) G(\varepsilon)=0,
$$

for inner edge $(\xi=\varepsilon)$,

and

$$
F(1) \frac{\mathrm{d}^{2} G(1)}{\mathrm{d} \xi^{2}}+\left(v_{\theta} F(1)+K_{r a}\right) \frac{\mathrm{d} G(1)}{\mathrm{d} \xi}-\left(\frac{n^{2} \pi^{2}}{\Phi^{2}}\right) v_{\theta} F(1) G(1)=0,
$$

for outer edge $(\xi=1)$,

where $K_{r b}=b k_{r b} / D_{r b}$ and $K_{r a}=a k_{r a} / D_{r b} . K_{r b}, K_{r a}$ are rotational flexibility parameters at inner and outer edge respectively, while $k_{r b}, k_{r a}$ are rotational stiffness constants at inner and outer edge respectively.

$2.2 \mathrm{~b}$ When circular edges are elastically restrained against translation: Shear force per unit length required to deflect translational spring (Lessia 1969),

$$
V_{r}=k_{t} w(r, \theta) \text {. }
$$

From the Kirchhoff-Kelvin resultant (Lekhnitskii 1968)

$$
V_{r}=N_{r}+(1 / r) \partial M_{r \theta} / \partial \theta
$$

where $N_{r}$ is transverse shear force per unit length and $M_{r \theta}$ is twisting moment per unit length. 
Substituting for $N_{r}$ and $M_{r \theta}$ from (Lekhnitskii 1968) in (7), the equations (6) and (7) give (after simplifications and normalization):

$$
\begin{aligned}
F(\varepsilon) & \frac{\mathrm{d}^{3} G(\varepsilon)}{\mathrm{d} \xi^{3}}+\left(\frac{\left(\frac{\varepsilon \mathrm{d} F(\varepsilon)}{\mathrm{d} \xi+F(\varepsilon)}\right)}{\varepsilon}\right) \frac{\mathrm{d}^{2} G(\varepsilon)}{\mathrm{d} \xi^{2}} \\
& +\left(\frac{\left(v_{\theta} \varepsilon \frac{\mathrm{d} F(\varepsilon)}{\mathrm{d} \xi}-\left(k^{2}+\left(v_{\theta}+4 D_{k r}\right)\left(\frac{n^{2} \pi^{2}}{\Phi^{2}}\right)\right) F(\varepsilon)\right)^{1}}{\varepsilon^{2}}\right) \frac{\mathrm{d} G(\varepsilon)}{\mathrm{d} \xi} \\
& +\left(\frac{\left(\frac{-v_{\theta} \varepsilon \mathrm{d} F(\varepsilon)}{\mathrm{d} \xi}+\left(v_{\theta}+k^{2}+4 D_{k r}\right)\left(\frac{n^{2} \pi^{2}}{\Phi^{2}}\right)^{1} F(\varepsilon)\right)}{\varepsilon^{3}+K_{t b}}\right) G(\varepsilon)=0
\end{aligned}
$$

for inner edge $(\xi=\varepsilon)$,

and

$$
\begin{aligned}
F(1) & \frac{\mathrm{d}^{3} G(1)}{\mathrm{d} \xi^{3}}+\left(\frac{\mathrm{d} F(1)}{\mathrm{d} \xi+F(1)}\right) \frac{\mathrm{d}^{2} G(1)}{\mathrm{d} \xi^{2}} \\
& +\left(v_{\theta} \frac{\mathrm{d} F(1)}{\mathrm{d} \xi}+\left(k^{2}+\left(v_{\theta}+4 D_{k r}\right)\left(\frac{n^{2} \pi^{2}}{\Phi^{2}}\right)\right)\right) F(1) \frac{\mathrm{d} G(1)}{\mathrm{d} \xi} \\
& +\left(-v_{\theta} \frac{\mathrm{d} F(1)}{\mathrm{d} \xi}-\left(v_{\theta}+k^{2}+4 D_{k r}\right)\left(\frac{n^{2} \pi^{2}}{\Phi^{2}}\right) F(1)-K_{t a}\right) G(1)=0,
\end{aligned}
$$

for outer edge $(\xi=1)$,

where $K_{t b}=b^{3} k_{t b} / D_{r b}$ and $K_{t a}=a^{3} k_{t a} / D_{r b} . K_{t b}, K_{t a}$ are translational flexibility parameters at inner and outer edge respectively, while $k_{t b}, k_{t a}$ are translational stiffness constants at inner and outer edge respectively.

\section{Method of solution}

An exact solution of equation of motion (1) along with the equations derived for boundary conditions, namely, (4), (5), (8) and (9) does not seem possible due to the presence of variable coefficients. In view of this, an approximate solution has been obtained by applying a collocation technique. For this purpose, the non-dimensional displacement function $G(\xi)$ is approximated as:

$$
G(\xi)=\sum_{i=1}^{m} A_{i}\left(1+\alpha_{i} \xi+\beta_{i} \xi^{2}+\gamma_{i} \xi^{3}+\delta_{i} \xi^{4}\right) \xi^{a i},
$$

where $a_{i}=i-1$ and $A_{i}, \alpha_{i}, \beta_{i}, \gamma_{i}$, and $\delta_{i}$ are unknown coefficients. The coefficients $\alpha_{i}, \beta_{i}, \gamma_{i}$, and $\delta_{i}$ have been obtained after substituting for $G(\xi)$ and its derivatives from (10) in the four boundary condition equations (4), (5), (8) and (9). After substituting $G(\xi)$ and its derivatives (with known values of these coefficients) in (1), the required frequency equations have been 
obtained. The frequency equations have been solved computationally taking Chebyshev points as the interpolating nodes in the range $(\varepsilon, 1)$ as given by Conte \& Boor $(1985)$ :

$$
\xi_{i}=((\varepsilon+1) / 2)+((\varepsilon-1) / 2) \cos [((2 i-1) / 2 m) \pi],
$$

where $i=1, \ldots \ldots, m$ and $\varepsilon<\xi<1$.

\section{Results and discussions}

Herein, a computer program has been developed. The frequency parameters of a polar orthotropic annular sector plate of parabolically varying thickness (PVT) when the straight (radial) edges are simply-supported and the curved edges are elastically restrained against translation and rotation have been evaluated for the first three modes of vibration considering a typical combination of plate parameters. Considering Poisson's ratio $v_{\theta}(=0 \cdot 3)$ and shear rigidity modulus $D_{k r}(=0 \cdot 35)$, all the results have been computed upto four decimal places taking the Chebyshev points as the interpolating nodes with total subintervals $m$ equal to 12 .

The results for free transverse vibrations of polar orthotropic PVT annular sector plates are not available in the literature. Hence, to ascertain the accuracy of the present results, the frequency parameters computed for a uniform thickness polar orthotropic annular sector plate with all edges simply-supported have been compared with the results obtained by Rubin (1975), Irie et al (1979), Ramaiah (1980) for radii ratio $\varepsilon(=0 \cdot 05)$, opening angle $\Phi(=$ 100 degree $)$ and Poisson's ratio $v_{\theta}(=0 \cdot 3)$ and shown in table 1.

Table 1. Comparison of frequency parameter $\Omega$ of polar orthtropic sector plate when radial and circular edges are simply-supported; $\Phi=100$ degree, $\varepsilon=0 \cdot 05, v_{\theta}=0 \cdot 3$.

\begin{tabular}{|c|c|c|c|c|c|c|}
\hline \multirow[b]{2}{*}{$\mathrm{P}$} & \multirow[b]{2}{*}{$D_{k r}$} & \multirow[b]{2}{*}{ Estimates from } & \multicolumn{4}{|c|}{ Number of nodal diameters and circles $(n, s)$} \\
\hline & & & $(1,0)$ & $(2,0)$ & $(3,0)$ & $(1,1)$ \\
\hline \multirow[t]{5}{*}{$0 \cdot 1$} & \multirow[t]{5}{*}{$0 \cdot 35$} & Present & $20 \cdot 1350$ & 35.4957 & 54.5953 & 61.4873 \\
\hline & & Ramaiah (1980) & $\{20 \cdot 148\}$ & $\{35 \cdot 503\}$ & $\{54.614\}$ & $\{61 \cdot 968\}$ \\
\hline & & & $(20 \cdot 142)$ & $(35.497)$ & $(54.595)$ & $(61.514)$ \\
\hline & & Irie et al (1979) & $20 \cdot 3$ & 35.9 & $55 \cdot 0$ & $61 \cdot 6$ \\
\hline & & Rubin (1975) & 20 & 36 & 55 & 62 \\
\hline \multirow[t]{5}{*}{$1 \cdot 0$} & \multirow[t]{5}{*}{$0 \cdot 35$} & Present & $23 \cdot 2171$ & $49 \cdot 7877$ & 84.6303 & $66 \cdot 1827$ \\
\hline & & Ramaiah (1980) & $\{23 \cdot 226\}$ & $\{49 \cdot 847\}$ & $\{84.730\}$ & $\{66 \cdot 849\}$ \\
\hline & & & $(23 \cdot 220)$ & $(49 \cdot 788)$ & $(84.631)$ & $(66 \cdot 203)$ \\
\hline & & Irie et al (1979) & 23.5 & 49.9 & 84.6 & $66 \cdot 5$ \\
\hline & & Rubin (1975) & 23 & 50 & 85 & 66 \\
\hline \multirow[t]{5}{*}{10} & \multirow[t]{5}{*}{$4 \cdot 85$} & Present & $56 \cdot 2754$ & $136 \cdot 5492$ & 243.7693 & $129 \cdot 6886$ \\
\hline & & Ramaiah (1980) & $\{56 \cdot 289\}$ & $\{136 \cdot 58\}$ & $\{244 \cdot 4\} 2$ & $\{130 \cdot 21\}$ \\
\hline & & & $(56 \cdot 276)$ & $(136 \cdot 55)$ & (243.77) & $(129 \cdot 71)$ \\
\hline & & Irie et al (1979) & $56 \cdot 8$ & $136 \cdot 7$ & $243 \cdot 8$ & $130 \cdot 9$ \\
\hline & & Rubin (1975) & 56 & 137 & 244 & 128 \\
\hline
\end{tabular}

Notes: $1 . n-1=$ number of radial nodal diameters;

2. $s=$ number of nodal circles;

3. Estimates in Irie et al (1979) are obtained by the Rayleigh-Ritz method with quintic spline polynomials as admissible functions, in Rubin (1975) by Forbenius method and in Ramaiah (1980) by the Rayleigh-Ritz method with simple polynomials as admissible functions taking number of terms 5 in parentheses \{\} and 8 in parentheses ( ) 
Frequency parameters $\Omega$ of polar orthotropic PVT annular sector plate with the radial edges simply supported and the circular edges elastically restrained against translation and rotation have been computed for the first three modes of vibration considering two separate sets of plate parameters. In the first set, taking radii ratio $\varepsilon(=0 \cdot 5)$, the opening angle $\Phi(=30)$ and the taper parameters $\alpha(= \pm 0 \cdot 3)$, the results have been computed for the rigidity ratios $P(=0.5,1.0$ and 3.0$)$ in order to consider the three cases of a stiffened plate - radially, isotropically and circumferentially stiffened respectively. In the second set, taking the radal ratio $\varepsilon(=0 \cdot 3)$, the opening angle $\Phi\left(=60^{\circ}\right)$ and the taper parameters $\alpha(= \pm 0 \cdot 5)$, the results have been computed only for the rigidity ratios $P(=0.5)$.

In first set, the four values, i.e. $\left(0,1,100\right.$ and $\left.10^{15}\right)$ for each of the flexibility parameters $\left(K_{t b}, K_{t a}, K_{r b}\right.$, and $\left.K_{r a}\right)$ have been considered. In the second set, the three values, i.e. $\left(0,1\right.$, and $\left.10^{15}\right)$ for each of the translational flexibility parameters and the five values, i.e. $(0$, $1,10,100,1000)$ for each of the rotational flexibility parameters have been considered. In both the cases, for simplicity, $\left(K_{t b}=K_{t a}\right)$ and $\left(K_{r b}=K_{r a}\right)$ have been considered and the numerical value $10^{15}$ has been assumed to be infinity $\left(_{* * *}\right)$.

During computations, in each set, for each given value of the translational flexibility parameters, all the given values of rotational flexibility parameters have been considered separately. The results computed for the first set have been shown in tables $2-4$. The results computed for the second set have been presented as plots in figures $2-4$, wherein the frequency

(a) Centrally Thicker Plate $(\alpha=-0.5)$ PVT

(b) Centrally Thinner Plate $(\alpha=0.5)$ PVT
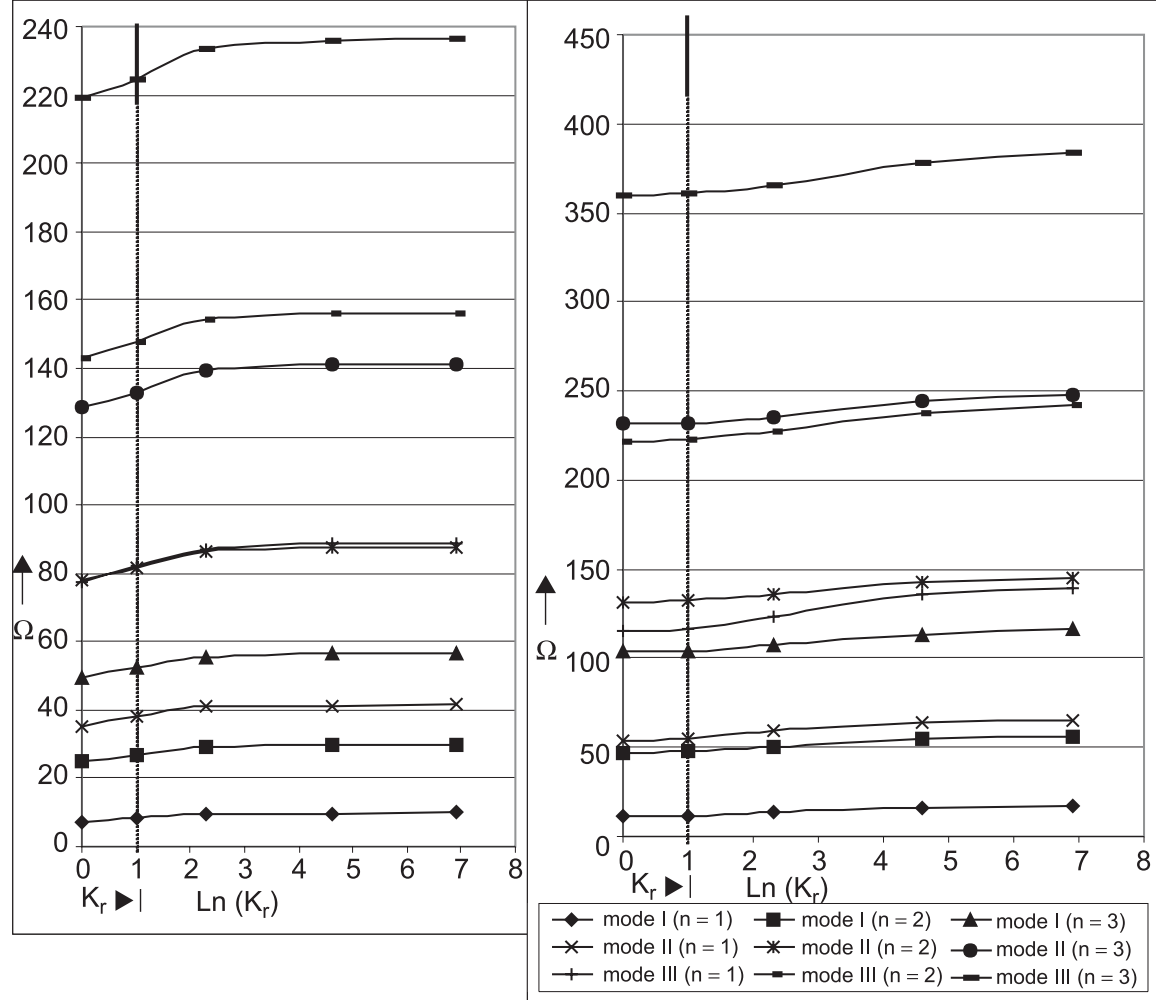

Figure 2. Frequency parameter $\Omega$ versus rotational flexibility parameters for a radially stiffened plate (a) when $\left(K_{r b}=K_{r a}=K_{r}\right)$ and (b) when $K_{t b}=K_{t a}=0\left(P=0 \cdot 5, \varepsilon=0 \cdot 3, \Psi=60^{\circ}\right)$. 
Table 2. Frequency parameter $\Omega$ of polar orthotropic radially and parabolically varying thickness sector plate when radial edges are simply-supported and circular edges are restrained elastically against translation and rotation; $\varepsilon=0 \cdot 5, \Phi=30^{\circ}$, first mode.

\begin{tabular}{|c|c|c|c|c|c|c|c|c|c|c|}
\hline & \multirow{2}{*}{$\begin{array}{c}K_{r b} \\
\& \\
K_{r a}\end{array}$} & \multicolumn{3}{|c|}{$P=0.5(n, s)$} & \multicolumn{3}{|c|}{$P=1.0(n, s)$} & \multicolumn{3}{|c|}{$P=3 \cdot 0(n, s)$} \\
\hline & & $(1,0)$ & $(2,0)$ & $(3,0)$ & $(1,0)$ & $(2,0)$ & $(3,0)$ & $(1,0)$ & $(2,0)$ & $(3,0)$ \\
\hline
\end{tabular}

$\begin{array}{llllll}0 & 0 & 29.3337 & 101.0490 & 211.2755\end{array}$

$\begin{array}{lllll}1 & 30.5477 & 102.8819 & 213.3953\end{array}$

$\begin{array}{llll}100 \quad 33.5899 & 111.3641 & 227.1340\end{array}$

*** $\quad 33.6988 \quad 111.8644 \quad 228.2325$

$\begin{array}{llllll}1 & 0 & 29.1248 & 100.9674 & 211.2266\end{array}$

$\begin{array}{lllll}1 & 30.3607 & 102.8067 & 213 \cdot 3491\end{array}$

$\begin{array}{lllll}100 \quad 33.4563 & 111.3159 & 227.1043\end{array}$

*** $\quad 33.5671 \quad 111.8177 \quad 228.2039$

$\begin{array}{lllll}100 & 0 & 115.9543 & 92.1539 & 206.2377\end{array}$

$\begin{array}{lllll}1 & 8.4171 & 94.7460 & 208.6482\end{array}$

$\begin{array}{llllll}100 & 11.0353 & 106.3360 & 224.1098\end{array}$

*** $\quad 12.2306 \quad 106.9987 \quad 225.3332$

$\begin{array}{lllllll}* * * & 0 & 75.8310 & 180.6149 & 334.0266\end{array}$

$\begin{array}{lllll}1 & 78.9840 & 182.7024 & 336.0370\end{array}$

$\begin{array}{llll}100 & 101.1235 \quad 201.0846 \quad 357.9517\end{array}$

$\begin{array}{lllll}* * * & 103.0968 & 203.1002 & 360 \cdot 8365\end{array}$

$\alpha=0 \cdot 3$

$\begin{array}{llllll}0 & 0 & 42.3896 & 160.6617 & 348.6429\end{array}$

$\begin{array}{lllll}1 & 42.8239 & 161.2021 & 349.2198\end{array}$

$\begin{array}{lllll}100 & 48.4790 & 172.6356 & 365.6119\end{array}$

$\begin{array}{lllll}* * * & 49.3240 & 175.8328 & 372.3514\end{array}$

$\begin{array}{llllll}1 & 0 & 42.3027 & 160.6354 & 348.6282\end{array}$

$\begin{array}{lllll}1 & 42.7391 & 161.1763 & 349.2054\end{array}$

$100 \quad 48.4176 \quad 172.6183 \quad 365.6016$

*** $\quad 49.2654 \quad 175.8176 \quad 372.3428$

$\begin{array}{lllll}100 & 0 & 28.8335 & 157.9849 & 347.1746\end{array}$

$\begin{array}{lllll}1 & 30.5564 & 158.5712 & 347.7676\end{array}$

$\begin{array}{lllll}100 \quad 41.2749 & 170.8806 & 364.5904\end{array}$

*** $\quad 42.5186 \quad 174.2945 \quad 371.4913$
$37.7936 \quad 133.4186 \quad 283.0663$ $39.3422 \quad 135.4062 \quad 285.3214$ $\begin{array}{llll}43.5368 & 144.9691 \quad 300.4341\end{array}$ $43.6948 \quad 145.5513 \quad 301.6782$

$\begin{array}{llll}37.6223 & 133.3471 \quad 283.0235\end{array}$ $39.1919135 .3401 \quad 285.2809$ $43.5958 \quad 145.5103 \quad 301.6530$

$\begin{array}{lll}59.9252 & 125.8169 & 278.6930\end{array}$ $\begin{array}{llll}15.0149 & 128.4098 \quad 281.1866\end{array}$ $31.1356 \quad 140.6022 \quad 297.7806$ $31.5964141 .3280 \quad 299.1360$ $\begin{array}{llll}43.4361 & 144.9267 & 300.4078\end{array}$

$\begin{array}{llll}56.2657 & 210 \cdot 0011 & 455.7887\end{array}$ $\begin{array}{lll}58.2807 & 212.4908 & 458.5622\end{array}$ $\begin{array}{llll}64.4222 & 225.3073 & 478.3181\end{array}$ $\begin{array}{llll}64.6790 & 226.1312 \quad 480.0322\end{array}$

$56.1236 \quad 209.9421 \quad 455.7536$ $\begin{array}{llll}58.1560 & 212.4360 & 458.5289\end{array}$ $\begin{array}{llll}64.3445 & 225.2729 & 478.2968\end{array}$ $\begin{array}{lll}64.6030 & 226.0979 \quad 480.0119\end{array}$

$\begin{array}{lll}37.6899 & 203.8854 & 452.2317\end{array}$ $\begin{array}{llll}42.6996 & 206.8271 & 455.1884\end{array}$ $\begin{array}{llll}55.7781 & 221.7993 & 476.1737\end{array}$ $56.2665 \quad 222.7496 \quad 477.9864$ $\begin{array}{llllll}83.9318 & 220.2940 & 419.1723 & 108.7769 & 317.4534 & 624.1257\end{array}$ $\begin{array}{llllll}86.8613 & 222.5295 & 421.5119 & 111.3657 & 320.1716 & 627.1145\end{array}$ $\begin{array}{lllllll}107.5997 & 242.1355 & 447.9128 & 129.5574 & 345.6539 & 663.7543\end{array}$ $\begin{array}{llllll}109.4580 & 244.2104 & 451.4673 & 131.1643 & 348.4510 & 669.0667\end{array}$

$\begin{array}{llllll}57.2423 & 218.8635 & 478.7061 & 90.2032 & 357.6280 & 793.4758\end{array}$ $\begin{array}{lllllll}57.7896 & 219.4292 & 479.2945 & 90.9257 & 358.3229 & 794.1798\end{array}$ $\begin{array}{lllllll}64.6108 & 231.7236 & 496.4201 & 100.6296 & 374.2479 & 815.5562\end{array}$ $\begin{array}{llllll}65.6447 & 235.2804 & 503.6838 & 102.2270 & 379.1373 & 825.1193\end{array}$

$\begin{array}{llllll}57.1803 & 218.8415 & 478.6937 & 90.1553 & 357.6105 & 793.4658\end{array}$ $\begin{array}{llllll}57.7292 & 219.4076 & 479.2823 & 90.8792 & 358.3056 & 794.1700\end{array}$ $\begin{array}{llllll}64.5695 & 231.7090 & 496.4114 & 100.6014 & 374.2363 & 815.5492\end{array}$ $\begin{array}{lllllll}65.6059 & 235.2677 & 503.6765 & 102.2015 & 379.1273 & 825.1135\end{array}$

$\begin{array}{llllll}50.3798 & 216.6388 & 477.4602 & 85.1573 & 355.8591 & 792.4803\end{array}$ $\begin{array}{llllll}51.1265 & 217.2396 & 478.0615 & 86.0435 & 356.5803 & 793.1941\end{array}$ $\begin{array}{llllll}60.2043 & 230.2521 & 495.5435 & 97.7350 & 373.0851 & 814.8569\end{array}$ $\begin{array}{llllll}61.5192 & 233.9982 & 502.9467 & 99.6178 & 378.1394 & 824.5396\end{array}$

$\begin{array}{lllllllllll}\text { *** } & 0 & 110.3404 & 270.9235 & 514.7065 & 122.5846 & 334.8887 & 658.0670 & 161.1270 & 497.1762 & 1009.6384\end{array}$ $\begin{array}{lllllllllll}1 & 111.8314 & 271.5523 & 515.1727 & 123.9046 & 335.4826 & 658.5960 & 162.1112 & 497.8486 & 1010.3130\end{array}$ $\begin{array}{llllllllll}100 & 142.1982 & 291.5219 & 533.8854 & 152.1102 & 355.3162 & 680.4216 & 185.8753 & 521.8274 & 1039.7339\end{array}$ $\begin{array}{lllllllllll}* * * & 151.3720 & 301.2387 & 546.6065 & 160.9365 & 365.3743 & 695.7841 & 193.9854 & 534.9201 & 1062.1994\end{array}$ 
Table 3. Frequency parameter $\Omega$ of polar orthotropic radially and parabolically varying thickness sector plate when radial edges are simply-supported and circular edges are elastically restrained against translation and rotation; $\varepsilon=0 \cdot 5, F=30^{\circ}$, second mode.

\begin{tabular}{|c|c|c|c|c|c|c|c|c|c|c|}
\hline \multirow{2}{*}{$\begin{array}{l}K_{t b} \\
\& \\
K_{t a}\end{array}$} & \multirow{2}{*}{$\begin{array}{c}K_{r b} \\
\& \\
K_{r a}\end{array}$} & \multicolumn{3}{|c|}{$P=0.5(n, s)$} & \multicolumn{3}{|c|}{$P=1 \cdot 0(n, s)$} & \multicolumn{3}{|c|}{$P=3 \cdot 0(n, s)$} \\
\hline & & $(1,2)$ & $(2,2)$ & $(3,2)$ & $(1,2)$ & $(2,2)$ & $(3,2)$ & $(1,2)$ & $(2,2)$ & $(3,2)$ \\
\hline
\end{tabular}

$\alpha=-0 \cdot 3$

$\begin{array}{lllllllllll}0 & 0 & 78.3438 & 219.5960 & 402.6536 & 97.8717 & 266.0546 & 491.9040 & 132.5476 & 367.1829 & 703.5882\end{array}$

$\begin{array}{lllllllllll}1 & 81.0980 & 221.8815 & 405.0374 & 99.8310 & 268.8397 & 494.7510 & 135.7398 & 370.8485 & 707.4191\end{array}$

$\begin{array}{llllllllll}100 & 91.4116 & 234.0347 & 421.9223 & 107.3115 & 283.8066 & 515.5372 & 147.2146 & 392.4252 & 737.4127\end{array}$

*** $\quad 91.9107 \quad 234.8295 \quad 423.3635 \quad 107.6788 \quad 284.7814 \quad 517.3516 \quad 147.7279 \quad 393.9372 \quad 740.1758$

$\begin{array}{lllllllllll}1 & 0 & 78.0007 & 219.5535 & 402.6392 & 97.7127 & 266.0344 & 491.8920 & 132.5099 & 367.1677 & 703.5787\end{array}$

$\begin{array}{llllllllllll}1 & 80.8000 & 221.8420 & 405.0237 & 99.6765 & 268.8207 & 494.7395 & 135.7027 & 370.8343 & 707.4100\end{array}$

$\begin{array}{lllllllllll}100 & 91.2450 & 234.0114 & 421.9142 & 107.1936 & 283.7950 & 515.5307 & 147.1761 & 392.4177 & 737.4078\end{array}$

$\begin{array}{lllllllllll}* * * & 91.7493 & 234.8073 & 423.3558 & 107.5633 & 284.7703 & 517.3455 & 147.6891 & 393.9302 & 740.1715\end{array}$

$\begin{array}{lllllllllll}100 & 0 & 257.1012 & 214.6461 & 401.2149 & 126.5835 & 264.0565 & 490.7031 & 128.7338 & 365.6843 & 702.6428\end{array}$

$\begin{array}{llllllllllll}1 & 126.6470 & 217.3873 & 403.6729 & 69.3760 & 266.9608 & 493.6125 & 131.8223 & 369.4464 & 706.5205\end{array}$

$\begin{array}{lllllllllll}100 & 69.6973 & 231.6187 & 421.1075 & 92.5146 & 282.6496 & 514.8822 & 142.9050 & 391.6813 & 736.9245\end{array}$

*** $\quad 71.2816 \quad 232.5355 \quad 422.5925 \quad 93.3972 \quad 283.6731 \quad 516.7380 \quad 143.4026 \quad 393.2398 \quad 739.7245$

$\begin{array}{llllllllllll}* * * & 0 & 183.1088 & 319.4701 & 521.8988 & 188.3521 & 360.7725 & 621.0736 & 208.2310 & 477.1539 & 862.6432\end{array}$

$\begin{array}{llllllllllll}1 & 188.2758 & 322.4957 & 524.2082 & 193.3263 & 363.4325 & 623.4006 & 212.5355 & 479.5832 & 865.2437\end{array}$

$\begin{array}{llllllllllll}100 & 236.3626 & 358.0473 & 555.3204 & 240.1360 & 395.1013 & 655.3073 & 254.7706 & 509.1313 & 904.5691\end{array}$

$\begin{array}{llllllllll}* * * & 241.7752 & 363.0998 & 560.3857 & 245.4432 & 399.5965 & 660.4311 & 259.6920 & 513.2269 & 911.4183\end{array}$

$\alpha=0 \cdot 3$

$\begin{array}{lllllllllll}0 & 0 & 105.8689 & 309.9285 & 597.2560 & 131.9379 & 389.5167 & 747.6496 & 186.7532 & 556.6228 & 1106.0771\end{array}$

$1 \quad \begin{array}{llllllllll}0 & 107.7570 & 310.9679 & 597.8451 & 132.8453 & 390.1416 & 748.2752 & 187.6448 & 557.5399 & 1106.9336\end{array}$

$\begin{array}{lllllllllll}100 & 125.2852 & 326.7122 & 614.0824 & 145.6658 & 405.6341 & 767.5868 & 202.2387 & 580.7247 & 1134.6227\end{array}$

$\begin{array}{llllllllll}* * * & 127.8878 & 330.8106 & 621.0190 & 147.9814 & 410.5445 & 776.2811 & 205.0300 & 588.5008 & 1147.7707\end{array}$

$\begin{array}{lllllllllll}1 & 0 & 105.6284 & 309.8850 & 597.2511 & 131.8055 & 389.5082 & 747.6462 & 186.7312 & 556.6184 & 1106.0744\end{array}$

$1 \quad 107.5399 \quad 310.9280 \quad 597.8393 \quad 132.7204 \quad 390.1331 \quad 748.2719 \quad 187.6219 \quad 557.5356 \quad 1106.9310$

$\begin{array}{llllllllll}100 & 125.1705 & 326.6927 & 614.0793 & 145.5790 & 405.6274 & 767.5845 & 202.2068 & 580.7221 & 1134.6209\end{array}$

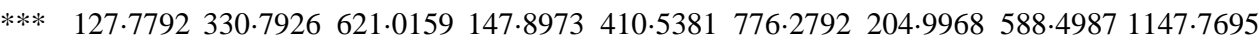

$\begin{array}{lllllllllll}100 & 0 & 68.5859 & 304.9074 & 596.5912 & 112.5149 & 388.6428 & 747.3099 & 184.2293 & 556.1884 & 1105.8159\end{array}$

$\begin{array}{lllllllllll}1 & 75.7541 & 306.4325 & 597.2055 & 115.2942 & 389.2758 & 747.9387 & 185.0197 & 557.1110 & 1106.6748\end{array}$

$\begin{array}{llllllllll}100 & 112.2620 & 324.6884 & 613.7005 & 135.8844 & 404.9469 & 767.3545 & 198.7447 & 580.4585 & 1134.4508\end{array}$

*** $\quad 115.7582 \quad 328.9568 \quad 620.6938 \quad 138.6334 \quad 409.9017 \quad 776.0960 \quad 201.4155 \quad 588.29201147 .6419$

*** $\quad 0 \quad 260.7057 \quad 456.0746 \quad 753.5494 \quad 267.8601 \quad 514.3525 \quad 906.7019 \quad 295.0429 \quad 690.44101297 .3498$

$\begin{array}{llllllllllll}0 & 263.4513 & 457.3869 & 754.2541 & 270.5022 & 515.3965 & 907.2870 & 297.3165 & 691.1040 & 1297.9492\end{array}$

$\begin{array}{llllllllllll}100 & 325.1900 & 499.0036 & 783.8511 & 330.6183 & 551.2268 & 934.0335 & 351.6167 & 718.7510 & 1327.7769\end{array}$

*** $\quad 348.4480 \quad 521.5205 \quad 806.3611 \quad 353.5679 \quad 572.1153 \quad 956.1242 \quad 373.4471 \quad 737.7812 \quad 1355.4043$ 
Table 4. Frequency parameter $\Omega$ of polar orthotropic radially and parabolically varying thickness sector plate when radial edges are simply-supported and circular edges are elastically restrained against translation and rotation; $\varepsilon=0 \cdot 5, F=30^{\circ}$, third mode.

\begin{tabular}{|c|c|c|c|c|c|c|c|c|c|c|}
\hline \multirow{2}{*}{$\begin{array}{l}K_{t b} \\
\& \\
K_{t a}\end{array}$} & \multirow{2}{*}{$\begin{array}{c}K_{r b} \\
\& \\
K_{r a}\end{array}$} & \multicolumn{3}{|c|}{$P=0.5(n, s)$} & \multicolumn{3}{|c|}{$P=1.0(n, s)$} & \multicolumn{3}{|c|}{$P=3 \cdot 0(n, s)$} \\
\hline & & $(1,1)$ & $(2,1)$ & $(3,1)$ & $(1,1)$ & $(2,1)$ & $(3,1)$ & $(1,1)$ & $(2,1)$ & $(3,1)$ \\
\hline
\end{tabular}

$\alpha=-0 \cdot 3$

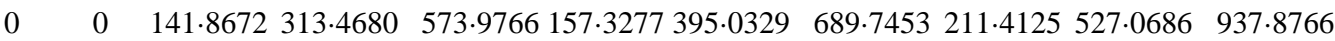

$\begin{array}{llllllllll}1 & 150.6436 & 319.2671 & 577.3227 & 164.4570 & 397.8356 & 692.9860 & 214.5029 & 530.8819 & 941.8296\end{array}$

$100 \quad 187.4506 \quad 351.2300 \quad 602.4563 \quad 196.2352 \quad 416.1724 \quad 719.1697 \quad 231.6887 \quad 557.2840 \quad 977.0614$

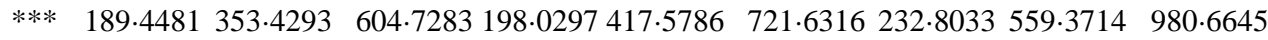

$\begin{array}{lllllllllll}1 & 0 & 141.5603 & 313.3152 & 573.9472 & 157.0239 & 394.9923 & 689.7376 & 211.2634 & 527.0599 & 937.8710\end{array}$

$\begin{array}{lllllllllll}1 & 150.3811 & 319.1347 & 577.2966 & 164.1985 & 397.7965 & 692.9788 & 214.3604 & 530.8736 & 941.8243\end{array}$

$100 \quad 187.3353 \quad 351.1789 \quad 602.4450 \quad 196 \cdot 1196416.1463 \quad 719.1656 \quad 231.5910 \quad 557.2791 \quad 977.0581$

$\begin{array}{llllllllll}* * * & 189.3390 & 353.3821 & 604.7182 & 197.9201 & 417.5537 & 721.6278 & 232.7086 & 559.3668 & 980.6637\end{array}$

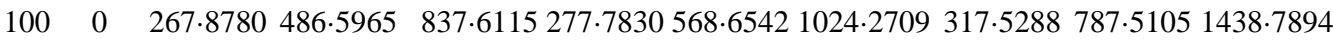

$\begin{array}{llllllllll}1 & 269.7211 & 304.2930 & 574.5259 & 137.6293 & 393.4423 & 692.2568 & 196.5045 & 530.0572 & 941.2902\end{array}$

$\begin{array}{llllllllll}100 & 175.9806 & 345.8927 & 601.3280 & 184.4982 & 413.4502 & 718.7393 & 221.1963 & 556.7959 & 976.7764\end{array}$

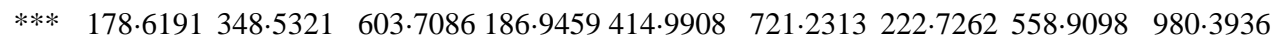

*** $\quad 0 \quad 348.5886 \quad 500.7179 \quad 731.7919351 .7713533 .9273 \quad 833.3447 \quad 364.3972 \quad 649.8608 \quad 1105.4564$

$1 \quad 355.0249 \quad 504.9913 \quad 734.7197358 .1332537 .7554 \quad 835.8766 \quad 370.4637 \quad 652.61841107 .9313$

$100 \quad 430.5224 \quad 564.5678 \quad 781.8899432 .9450592 .2819 \quad 877.1721 \quad 442.5623 \quad 694.01851150 .5048$

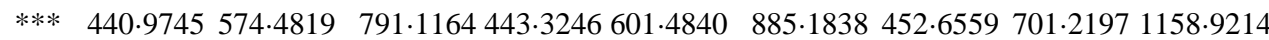

$\alpha=0 \cdot 3$

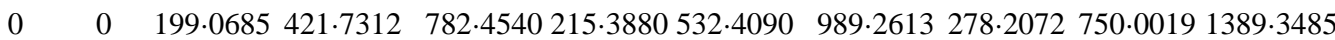

$1 \quad 204.4516 \quad 426.3621 \quad 785.8402 \quad 220.0493534 .0227 \quad 989.9530 \quad 280.1546 \quad 750.9271 \quad 1390.2545$

$100 \quad 258.9290 \quad 476.6289 \quad 826.6404269 .2607560 .85521013 .0899 \quad 310.6296 \quad 777.44131422 .1061$

*** $\quad 267.9451 \quad 486.6316 \quad 837.6228277 .8508568 .6791 \quad 1024.2731317 .5938 \quad 787.51391438 .7900$

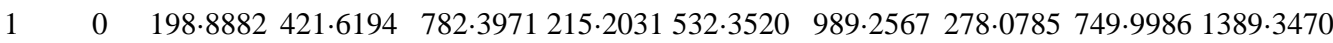

$1 \quad 204.2840 \quad 426.2589 \quad 785.7935 \quad 219.8793 \quad 533.9696 \quad 989.9487 \quad 280.0333 \quad 750.92391390 .2529$

$100 \quad 258.8543 \quad 476.5887 \quad 826.6248 \quad 269.1854 \quad 560.8282 \quad 1013.0866 \quad 310.5596 \quad 777.43791422 .1050$

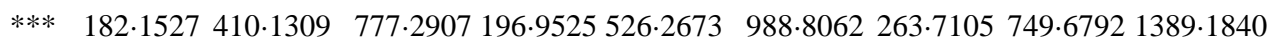

$\begin{array}{lllllllllll}100 & 0 & 456.4678 & 295.9619 & 570 \cdot 8274 & 263.0411 & 390.4035 & 688.9862 & 191.7641 & 526.2078 & 937.3163\end{array}$

$1 \quad \begin{array}{lllllllllll}0 & 188.3946 & 415.6277 & 781.1873 & 202.9324 & 528.3167 & 989.5064 & 266.6280 & 750.6006 & 1390.0911\end{array}$

$100 \quad 251.4913 \quad 472.5192 \quad 825.2713 \quad 261.7133 \quad 558.0792 \quad 1012.7687 \quad 303.4227 \quad 777.09191421 .9955$

*** $\quad 261.2820483 .0619 \quad 836.4782$ 271.0786 566.1409 $1023.9826310 .9577 \quad 787.16501438 .7111$

*** $\quad 0 \quad 496.2645 \quad 710.16231036 .7315500 .4841752 .97901177 .0833 \quad 517.1508 \quad 908.2153 \quad 1590.4836$

$1 \quad 499.7494712 .3002 \quad 1037.9578503 .9329754 .89531177 .9893 \quad 520.4577 \quad 909.42891591 .0599$

$100 \quad 590.5311 \quad 780.70751087 .2415593 .9248 \quad 817.83101218 .0312 \quad 607.3667 \quad 955.31591622 .5335$

*** $\quad 632.5488 \quad 821.22721125 .9937635 .7391856 .53031253 .0114648 .3900988 .33671655 .8002$ 
(a) Centrally Thicker Plate $(\alpha=-0.5)$ PVT

(b) Centrally Thinner Plate $(\alpha=0.5)$ PVT
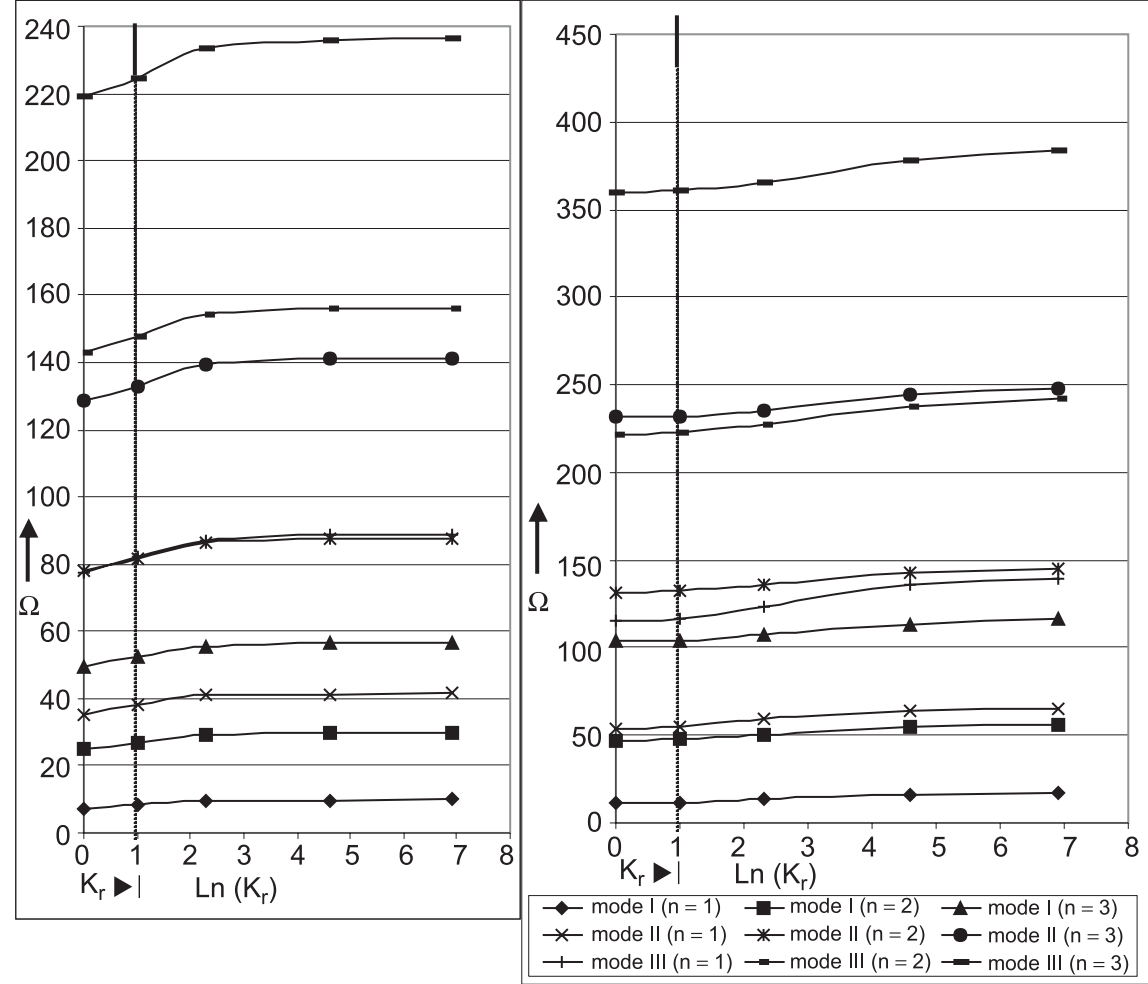

Figure 3. Frequency parameter $\Omega$ versus rotational parameters for a radially stiffened plate (a) when $\left(K_{r b}=K_{r a}=K_{r}\right)$ and (b) when $K_{t b}=K_{t a}=1\left(P=0 \cdot 5, \varepsilon=0 \cdot 3, \Psi=60^{\circ}\right)$.

parameters versus rotational flexibility parameters curves have been plotted considering a reduced scale for the rotational flexibility parameters by taking their logarithmic values after unity.

From the tables and the curves plotted in the figures, it can be noted that for each given value of the translational flexibility parameters the numerical value of a frequency parameter computed for a centrally thinner plate ( $\alpha=0 \cdot 3,0 \cdot 5)$, in most of the cases, is higher than its corresponding value computed for a centrally thicker plate $(\alpha=-0 \cdot 3,-0.5)$ in each mode of vibration of the all considered rigidity ratios except a few results where the trend is reverse.

See, for examples, in tables 2 to 4 when $K_{t b}=K_{t a}=100$ and

$$
\begin{aligned}
& K_{r b}=K_{r a}=0 ; n=1, \text { for } P=0 \cdot 5 \text { and } 1 \cdot 0, \text { (first mode) } \\
& K_{r b}=K_{r a}=0 ; n=1 \text { for } P=0 \cdot 5 \text { and } 1 \cdot 0, \text { (second mode) } \\
& K_{r b}=K_{r a}=1 ; n=1 \text { for } P=0 \cdot 5,(\text { second mode) } \\
& K_{r b}=K_{r a}=0 ; n=1,2,3 \text { for } P=1 \cdot 0 \text { and } 3 \cdot 0, \text { (third mode) } \\
& K_{r b}=K_{r a}=1 ; n=1 \text { for } P=0 \cdot 5,(\text { third mode) } .
\end{aligned}
$$

It should also be noted that for each given value of translational flexibility parameter, a frequency parameter, in most of the cases, increases with the increase of rotational flexibility 
(a) Centrally Thicker Plate $(\alpha=-0.5)$ PVT

(b) Centrally Thinner Plate $(\alpha=0.5)$ PVT

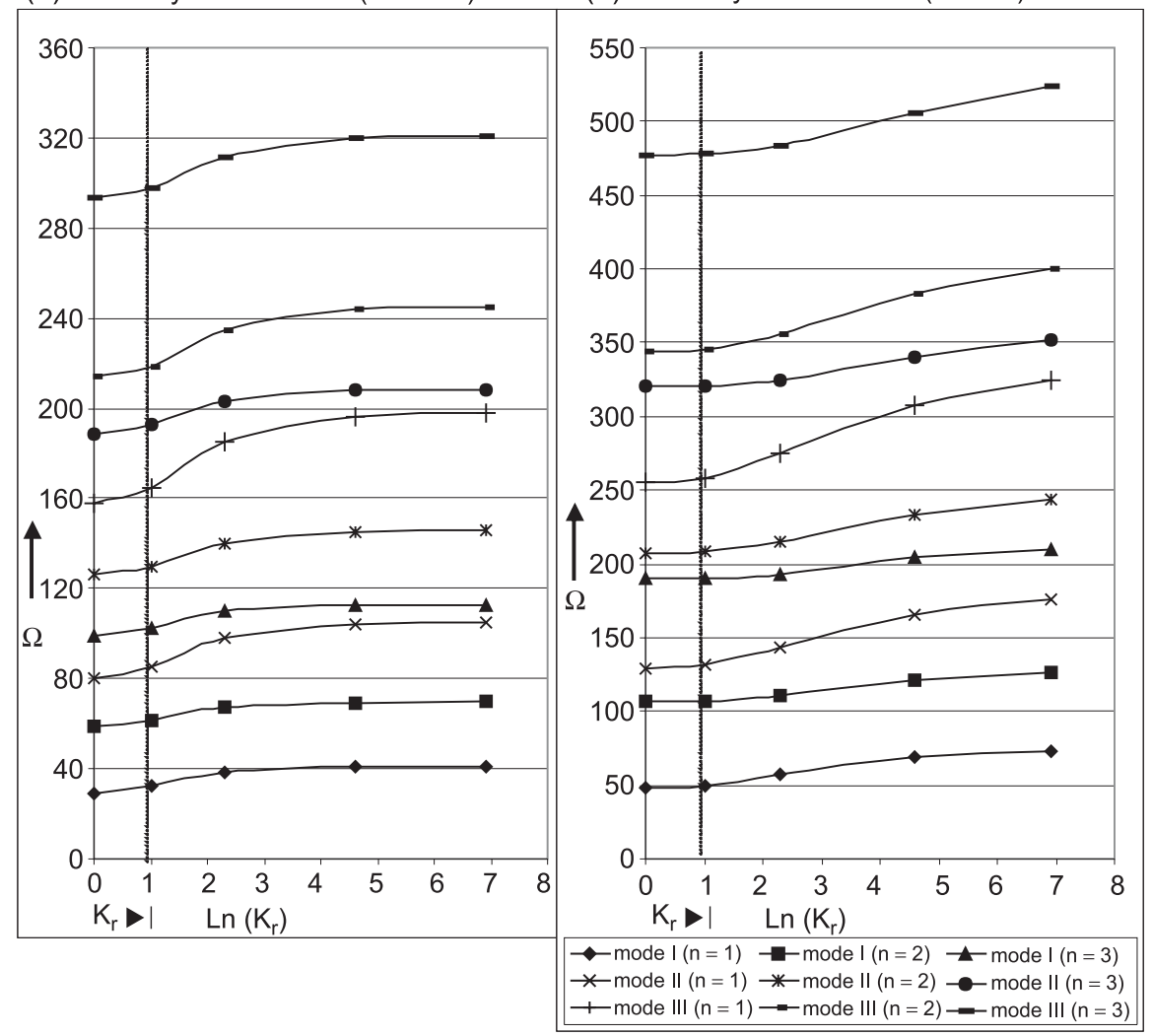

Figure 4. Frequency parameter $\Omega$ versus rotational flexibility parameters for a radially stiffened plate (a) when $\left(K_{r b}=K_{r a}=K_{r}\right)$ and (b) when $K_{t b}=K_{t a}=* * *\left(P=0 \cdot 5, \varepsilon=0 \cdot 3, \Psi=60^{\circ}\right)$.

parameters in each mode of vibration both for the centrally thinner as well as for the centrally thicker plates except a few results for $K_{t b}=K_{t a}=100$ (see tables 2 to 4).

From figures $2-4$, it is seen that, when the value of the rotational flexibility parameters is more than 100 , the variation in the value of the frequency parameter, in each mode of vibration, seems to be greater for a centrally thinner plate in comparison to that for a centrally thicker plate but it is just the opposite when the value of the rotational flexibility parameters is in between 0 and 1 .

\section{Conclusions}

Comprehensive sets of numerical results of asymmetric vibration of polar orthotropic annular sector plate of parabolically varying thickness have been obtained. The straight (radial) edges are simply-supported while the curved edges are elastically restrained against translation and rotation and the first three modes of vibration have been considered for both centrally thinner and thicker cases of each type of stiffened plate. The results obtained in this note are expected to be useful for the designers and the researchers working in this field. 


\section{List of symbols}

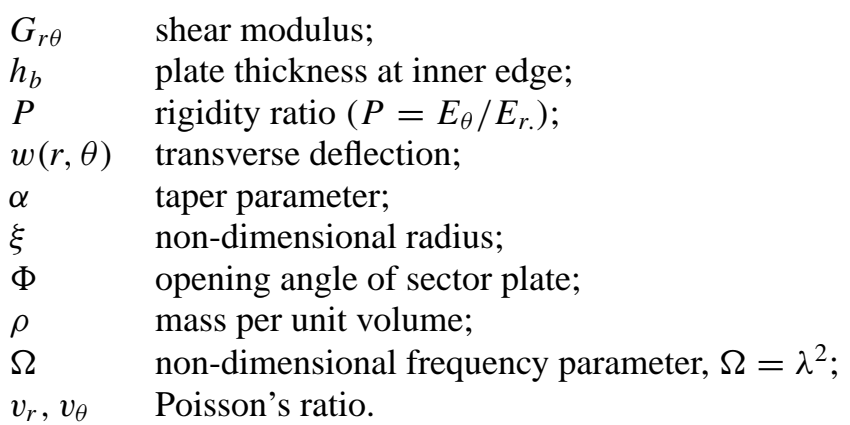

\section{References}

Ben-Amoz M 1959 Note on deflections and flexural vibrations of clamped sectorial plates. J. Appl. Mech. 26: 136-137

Bhattacharya A P, Bhowmic K N 1975 Free vibration of a sectorial plate. J. Sound Vibr. 41: 503-505

Chen D Y 1997 Axisymmetric vibration of circular and annular plates with arbitrarily varying thickness. J. Sound Vibr. 206: 114-121

Conte, Boor 1985 Elementary numerical analysis (an algorithmic approach) 3rd edn (New York: Mc Graw-Hill)

Houmat A 2001 A sector Fourier $p$-element applied to free vibration analysis of sectorial plates. $J$. Sound Vibr. 243: 269-282

Irie T, Yamada G, Ito F 1979 Free vibration of polar orthotropic sector plates. J. Sound Vibr 67: $89-100$

Irie T, Tanaka K, Yamada G 1988 Free vibration of a cantilever annular sector plate with curved radial edges. J. Sound Vibr. 122: 69-78

Kim C S, Dickinson S M 1990 The flectural vibration of the isotropic and polar orthotropic annular and circular plates with elastically restrained peripheries. J. Sound Vibr. 143: 171-179

Lekhnitskii S G 1968 Anisotropic plates (New York: Gordon \& Breach)

Lessia A W 1969 Vibration of plates. NASA SP 160

Lal R, Gupta U S 1982 Axisymmetric vibration of polar orthotropic annular plates of variable thickness. J. Sound Vibr. 83: 229-240

Liew K M, Liu F L 2000 Differential quadrature method for vibration analysis of shear deformable annular sector plates. J. Sound Vibr. 230: 335-356

Mukhopadhayay M 1979 A semi-analytic solution for free vibration of annular sector plates. J. Sound Vibr. 63: 87

Mukhopadhyay M 1982 Free vibration of annular sector plates with edges possessing different degrees of rotational restraint. J. Sound Vibr. 80: 275-279

Mizusawa T 1991 Application of spline element method to analyze vibration of annular sector plates. J. Sound Vibr. 149: 461-470

Narita Y 1985 Free vibration of polar-orthotropic sector plates resting on point supports. Trans. ASME

Ramaiah G K 1980 Flexural vibrations of polar orthotropic sector plates with simply supported straight edges. J. Sound Vibr. 70: 589-596

Ramaiah G K, Vijaykumar K 1974 Natural frequencies of circumferentially truncated sector plates with simply supported straight edges. J. Sound Vibr. 34: 53-61

Ramakrishnan R, Kununnasseril V X 1973 Free vibration of annular sector plates. J. Sound Vibr. 30: $127-129$ 
Rubin C 1957 Nodal circles and natural frequencies for the isotropic wedge. J. Sound Vibr. 39: $523-526$

Rubin C 1975 Vibrating modes for simply supported polar orthotropic sector plates. J. Acoust. Soc. Am. 58: 841-845

Rubin C 1978 Stability of polar orthotropic sector plates. J. Appl. Mech. 45: 448-450

Singh R P, Jain S K 2001 Asymmetric vibration of non-uniform circular annular plate by quintic spline collocation technique. Proc. 2nd Int. Conf. Theoretical, Applied, Computational and Experimental Mechanics

Westmann R A 1962 A note on free vibrations of triangular and sector plates. J. Aerosp. Sci. 29: $1139-1140$

Wilson J F, Garg D P 1978 Frequencies of annular plate and curved beams elements. AIAA J. 16: 270-272 